EPJ Web of Conferences 21, 09002 (2012)

DOI: $10.1051 /$ epjconf/20122109002

(C) Owned by the authors, published by EDP Sciences, 2012

\title{
Pre-equilibrium emission of $\alpha$-particles with energies down to the region of those from compound nucleus decay
}

\author{
A.A. Cowley ${ }^{1,2, \text { a }}$ \\ 1 Department of Physics, Stellenbosch University, Private Bag X1, Matieland 7602, South \\ Africa \\ 2 iThemba Laboratory for Accelerator Based Sciences, P O Box 722, Somerset West 7129, \\ South Africa
}

\begin{abstract}
At incident energies in the 100 to $200 \mathrm{MeV}$ range, nucleon-induced inclusive reactions with emission of light composite particles (such as $\alpha$-particles) into the continuum, display an enhanced yield which increases as the emission energy drops towards compound-nucleus values. It is postulated that a simple reaction mechanism, which clearly manifests itself in some $(p, p \alpha)$ knockout studies as sequential decay from inelastic excitation, should be investigated more carefully as a possible explanation of the observed phenomenon in inclusive studies.
\end{abstract}

\section{Introduction}

At projectile energies in the range of 100 to $200 \mathrm{MeV}$, nucleon-induced pre-equilibrium emission of intermediate-mass fragments from atomic nuclei presents a challenge to our understanding of the relevant physics [1]. In spite of considerable effort, unambiguous identification of the dominant reaction mechanisms leading to emission of composite ejectiles, such as $\alpha$ particles, remains elusive. It is significant that in the case of nucleon emission, a number of related and consistent theoretical approaches that address the same physics appear to provide a reasonable account of experimental data over a large incident energy and target-mass range $[2]$.

One of the most ambitious projects to provide simulations of nuclear reactions in the incident energy range up to $200 \mathrm{MeV}$ is TALYS [3], which combines the most reliable theoretical models in a single computer code package. The results from this code successfully reproduce a wide selection of experimental (N,Nx) pre-equilibrium data [4]. Nevertheless, when for example applied to ${ }^{3} \mathrm{He}$ and $\alpha$-particle emission, the results from the code seem to be very disappointing [5]. More specific, for the inclusive ${ }^{59} \mathrm{Co}(p, \alpha x)$ reaction at an incident energy of $160 \mathrm{MeV}$ Bevilacqua [5] finds that the TALYS default mix of nucleon transfer and knockout overestimates emission of these ejectiles at high emission energy by a disturbing margin, and underestimates it at low emission energies. Better, but still far from satisfactory agreement is obtained by turning off the knockout component in the code. However, this procedure exacerbates the problem at the low energy end. A similar problem at low $\alpha$-particle emission energies is encountered in the statistical multistep knockout analysis of Cowley et al. [6] of exactly the same experimental $(p, \alpha x)$ data. Yet another theoretical study [7] of comparable data of ${ }^{58} \mathrm{Ni}(p, \alpha x)$ at $175 \mathrm{MeV}$ suffers from essentially the same problem when only a coalescence mechanism is combined with the intranuclear cascade. In order to resolve the underestimation of $\alpha$-particle emission, Budzanowski et al. [7] postulate emission from a fast-moving source originating from breakup of the target nucleus.

a e-mail: aac@sun.ac.za

This is an Open Access article distributed under the terms of the Creative Commons Attribution-Noncommercial License 3.0, which permits unrestricted use, distribution, and reproduction in any noncommercial medium, provided the original work is properly cited. 


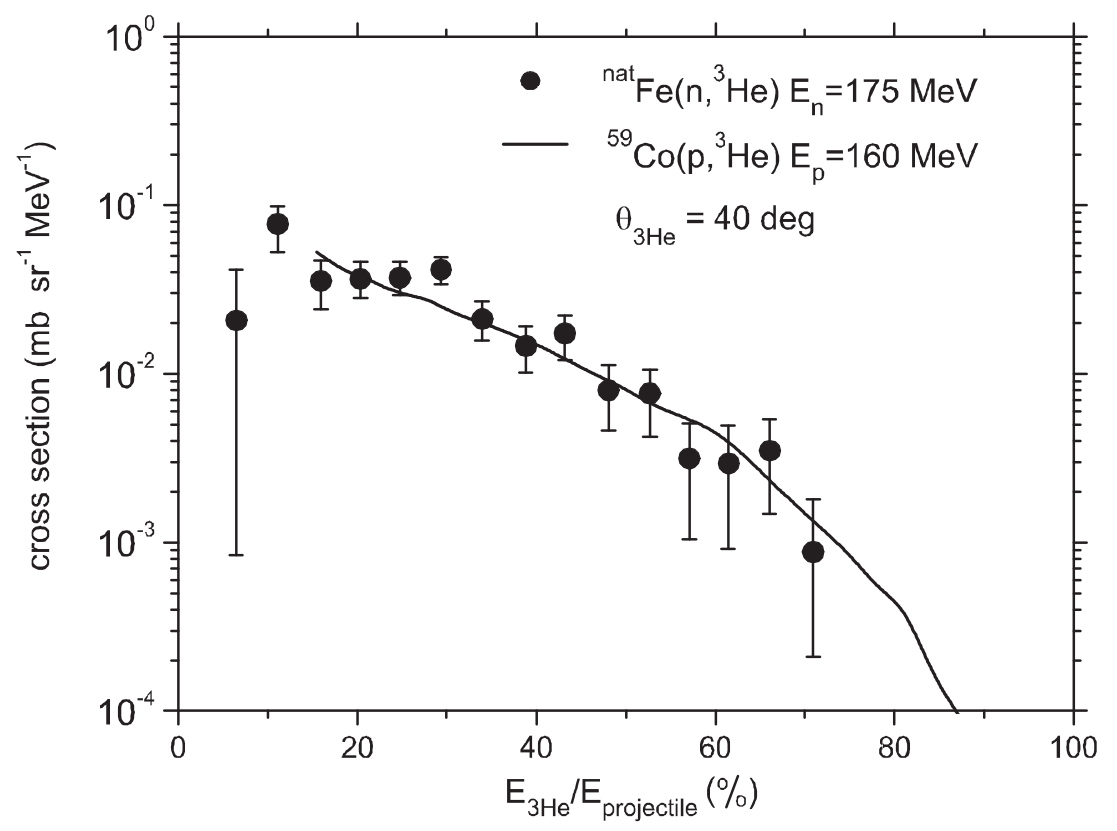

Fig. 1. Double differential cross section distribution for the reaction ${ }^{n a t} \mathrm{Fe}\left(n,{ }^{3} \mathrm{He} x\right)$ from Bevilacqua [5] (data points with error bars) compared with the results of Cowley et al. [8] for ${ }^{59} \mathrm{Co}\left(p,{ }^{3} \mathrm{He} x\right)$ at an incident energy of $160 \mathrm{MeV}$. For clarity of representation, the data of Ref. [8] is shown as a curve drawn through those experimental points. The statistical uncertainty associated with the solid line is less than the symbol size used to display the data of Bevilacqua [5]. To correct for the difference in incident energy, results are displayed as a function of the ratio of emitted ${ }^{3} \mathrm{He}$ energy to the incident energy of the projectile.

A well known problem with nucleon-induced pre-equilibrium reaction cross section distributions, which makes theoretical interpretations very difficult, is that in the region of interest to the present paper, they are fairly featureless and change rather slowly as a function of incident energy and target mass. Furthermore, due to an inevitable complicated reaction mechanism containing many components, and lack of knowledge regarding intermediate reaction products that lead to observed particle emission, an understanding of the complete reaction mechanism is difficult. As pointed out by Koning et al. [4], their two-component exciton treatment contains many ingredients which can not be directly verified independently. Unfortunately the result is that a deficiency in one part of a model can perhaps be compensated with an unrelated phenomenological feature.

Another property of particle emission in nucleon-induced pre-equilibrium reactions is that experimental results appear to scale with incident energy and target in a relatively simple way which is much less sensitive to details of the nuclear system than theory would suggest. As an example of this insensitivity, an emission energy distribution for the reaction ${ }^{59} \mathrm{Co}\left(p,{ }^{3} \mathrm{He} x\right)$ at an incident energy of $160 \mathrm{MeV}[8]$ is compared with experimental results at the same scattering angle for the reaction ${ }^{n a t} \mathrm{Fe}\left(n,{ }^{3} \mathrm{He} x\right)$ at an incident energy of $175 \mathrm{MeV}[5]$ in Fig.1. Cross sections are plotted as a function of the ratio of emission energy to incident energy. The observed correspondence between these two sets of data is remarkable, especially if we keep in mind that measurements with neutrons as projectiles present severe experimental challenges which could compromise the quality of the data [9] unless extreme care is taken.

Due to the widespread observed insensitivity, of which the quoted case in the previous paragraph is just one example, we assume in this paper that an effect observed for one, or a only a few, target nuclei is representative of a far wider range. Incident relative to emission 
energy dependence is assumed to scale as a simple ratio for estimation of their influence on data.

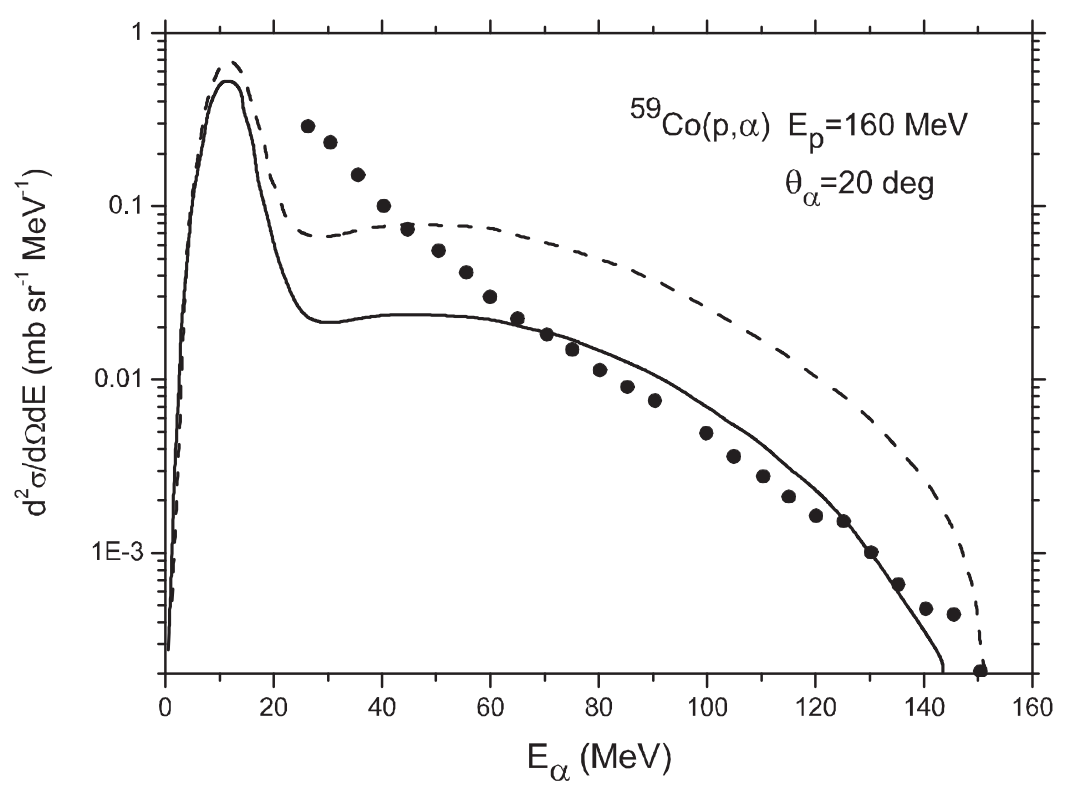

Fig. 2. Double differential cross section distribution for the ${ }^{59} \mathrm{Co}(p, \alpha x)$ reaction at an incident energy of $160 \mathrm{MeV}$. The experimental data of Cowley et al. [6] are shown with error bars which are smaller than the symbol size. The curves represent pre-equilibrium calculations by Bevilacqua [5] with default TALYS [3] input (dashed line) and with the knockout component switched off (solid line).

The main purpose of the present paper is to suggest a simple inelastic mechanism for the enhanced proton-induced $\alpha$-particle yield at energies down to values comparable to those associated with emission from the compound system. The justification for this hypothesis is that in some exclusive $(p, p \alpha)$ knockout studies [10-13], it is observed that low-lying states in the target system, which undergo sequential $\alpha$-particle decay, are inelastically excited. Although no explicit calculations are possible at this stage, additional experiments that could enable quantitative cross section calculations will be suggested. Note that the virtue of the proposed experiments and analysis does not depend on whether either a knockout or a nucleon transfer process drives the $\alpha$-particle emission at higher energies.

\section{Results and discussion}

In Fig. 2 a comparison is shown between TALYS calculations [5] and an experimental [6] ${ }^{59} \mathrm{Co}(p, p \alpha)$ double differential cross section emission energy distribution at an incident energy of $160 \mathrm{MeV}$ and a scattering angle of 20 degrees. The dashed line represents the default input to the TALYS code. The solid curve is a prediction for zero contribution from knockout; only nucleon transfer remains (apart from the cascade mechanism, of course, which does not contribute directly to $\alpha$-particle emission). Clearly the experimental $\alpha$-particle yield greatly exceeds the prediction at emission energies lower than about $60 \mathrm{MeV}$ when the upper part of the spectrum is correctly reproduced. This result is representative for those at somewhat larger angles. It is interesting to note that an alternative knockout analysis by Cowley et al. [6] displays the same qualitative underestimation at low $\alpha$-particle emission energies for the same 
data set. Although both interpretations can not be simultaneously correct, it is nevertheless significant that both reaction mechanisms agree on the underestimation.

A possible explanation for the discrepancy between theoretical treatment and experimental data is offered by explicit $(p, p \alpha)$ measurements on ${ }^{12} \mathrm{C}$ and ${ }^{40} \mathrm{Ca}$. For ${ }^{12} \mathrm{C}$ this is displayed in Fig.3, where distorted wave impulse approximation (DWIA) predictions are compared with energy sharing distributions at coplanar quasifree angles for the $(p, \alpha p)$ knockout reaction to the ground state. These cross sections, as a function of $\alpha$-particle energy, have been derived from results of Mabiala et al. [11] at an incident energy of $100 \mathrm{MeV}$. Based on a number of criteria it is safe to assume that the DWIA correctly estimates the contribution of direct knockout [11].

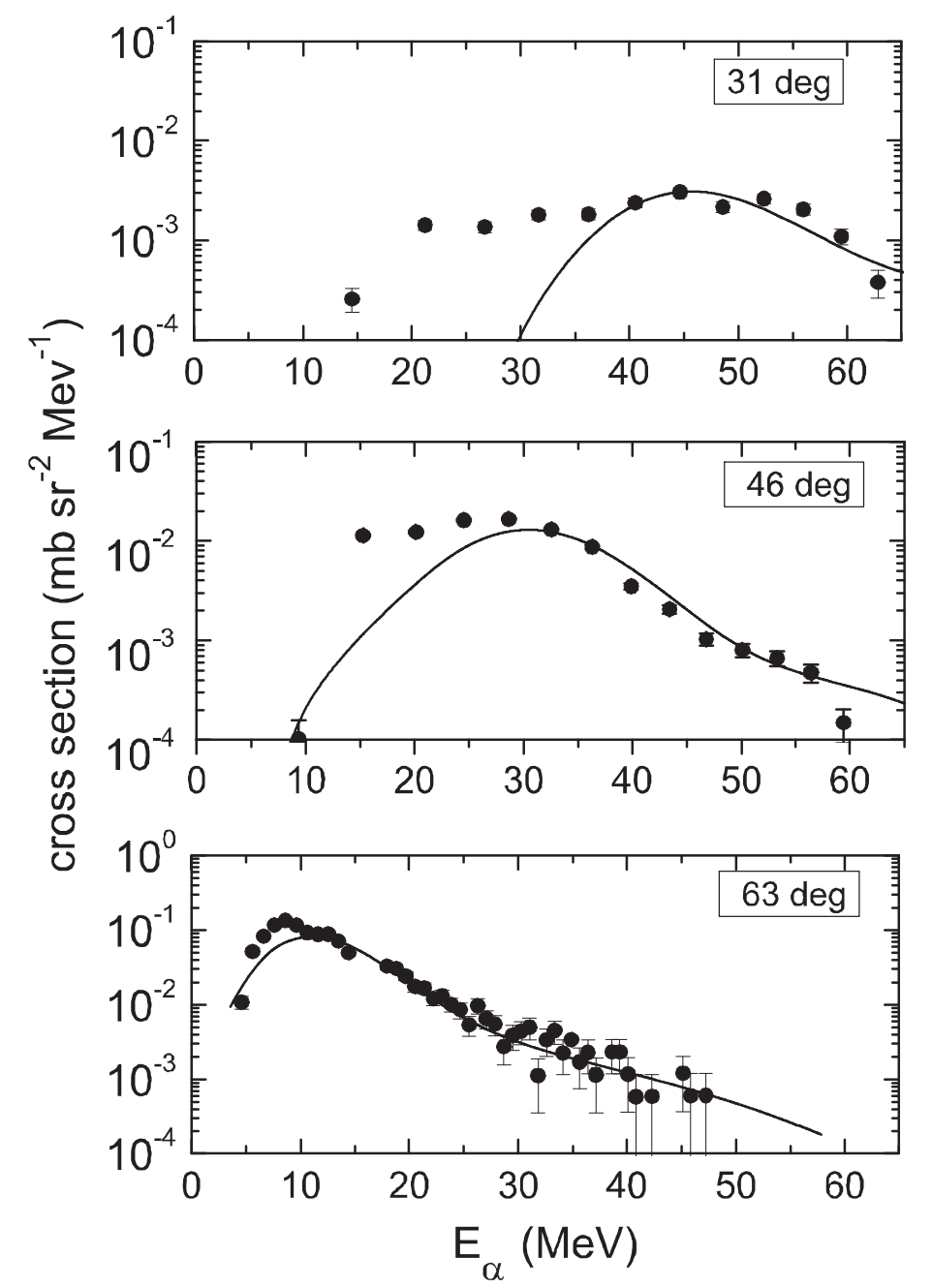

Fig. 3. Energy sharing cross section distribution for the ${ }^{12} \mathrm{C}(p, \alpha p)^{8} \mathrm{Be}$ knockout reaction at quasifree angles extracted from the study of Mabiala et al. [11] at an incident energy of $100 \mathrm{MeV}$. Results are shown as a function of $\alpha$-particle kinetic energy. Angles of observation of $\alpha$-particles are indicated. The curves represent results of DWIA predictions for a pure knockout reaction.

We find that there is an enhanced yield, compared to direct knockout, at the lower $\alpha$ particle energies at all angles. Closer investigation of the original data [11] reveals that this 
$\mathrm{CNR} * 11$

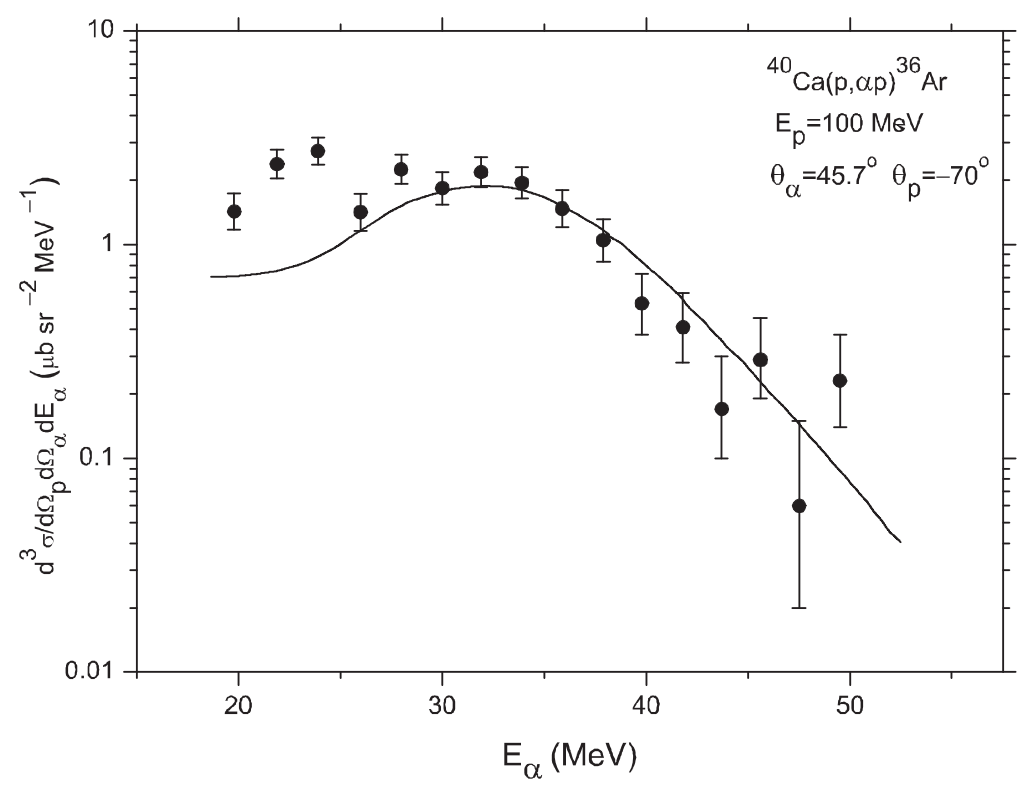

Fig. 4. Results for the ${ }^{40} \mathrm{Ca}(p, \alpha p)^{36} \mathrm{Ar}$ knockout reaction extracted from the data of Carey et al. [12] at an incident energy of $100 \mathrm{MeV}$. See caption to Fig. 2 for further details.

yield originates from $\alpha$-particle decay from excited states of the target nucleus. It appears that the phenomenon observed for ${ }^{12} \mathrm{C}$ applies also to a heavier nucleus such as ${ }^{40} \mathrm{Ca}$, as an adaptation of the results of Carey et al. [12] shows in Fig.4. This feature is also observed at higher incident energy, much beyond the $200 \mathrm{MeV}$ or so of interest to this investigation [13].

As was mentioned in the introduction, as an alternative interpretation, Budzanowski et al. [7] ascribe the additional yield of $\alpha$-particles in a related experiment to a quite different reaction mechanism. They apply their model, which evolved from successful applications at several $\mathrm{GeV}[14,15]$, to a proton incident energy of $175 \mathrm{MeV}$ on ${ }^{58} \mathrm{Ni}$. In this model, during the cascade phase from which emission also occurs, the excess of $\alpha$-particles mainly comes from a fast-moving source from breakup of the target, which co-exists with a slow-moving source and a fireball. Although intuitively attractive at projectile energies in the $\mathrm{GeV}$ range, it is not clear that such a mechanism should be expected to extrapolate smoothly to incident energies as low as $100 \mathrm{MeV}$.

The simplicity of our suggested reaction mechanism, together with its clear presence in actual knockout experiments, makes it a compelling candidate as a possible explanation of the observed phenomenon. However, a number of questions needs to be addressed before quantitative calculations for a specific reaction can be implemented. The most important issue is to investigate whether the sequential decay observed for the targets ${ }^{12} \mathrm{C}$ and ${ }^{40} \mathrm{Ca}$ is generally accessible to other nuclear species. Also, whereas the angular distribution of the knockout process as a function of proton scattering angle for a specific $\alpha$-particle angle is well predicted by the DWIA, the equivalent distribution for sequential decay still needs to be determined at the incident energy of interest.

It should be recalled that the relative cross section contribution of a knockout process to the continuum yield is undoubtedly much lower than the sequential decay, due to the much smaller solid angle available to knockout kinematics. Therefore it should be appreciated that Figs. 3 and 4 display distorted relative yields of the two coincidence cross sections when applied to a double differential spectrum. More specific, if we write the $(p, \alpha p)$ coincidence cross section 
for the residual nucleus in its ground state (corresponding to the data shown in Figs. 3 and 4) as

$$
\frac{d^{3} \sigma}{d \Omega_{\alpha} d \Omega_{p} d E_{\alpha}},
$$

where the quantities $d \Omega_{\alpha}$ and $d \Omega_{p}$ denote solid angles, then $d E_{\alpha}$ represents the differential kinetic energy of the $\alpha$-ejectile. Apart from a small amount of recoil energy of the residual nucleus, the available energy is shared by the two light ejectiles. In order to convert the cross section of the $(p, \alpha p)$ coincidence distribution to the double differential cross section for the $(p, \alpha)$ reaction, we need to determine the quantity

$$
\frac{d^{2} \sigma}{d \Omega_{\alpha} d E_{\alpha}}=\int \frac{d^{3} \sigma}{d \Omega_{\alpha} d \Omega_{p} d E_{\alpha}} d \Omega_{p}
$$

The sequential decay process is driven by an inelastic excitation, which at the incident energies of interest will vary more slowly in coordinate space than a quasifree knockout reaction, therefore the former reaction mechanism is expected to contribute even more strongly at the low emission energy than directly implied by the coincidence yield.

\section{Summary and conclusion}

It was shown that the intranuclear nucleon-nucleon cascade, which is an intrinsic mechanism driving the pre-equilibrium process in nucleon-induced reaction in the incident energy range of 100 to $200 \mathrm{MeV}$, generally appears to underestimate the formation of composite ejectiles, such as $\alpha$-particles. This phenomenon becomes increasingly more noticeable as the ejectile energy drops toward a value close to emission from the compound nucleus. The observed deficiency may be resolved by invoking exotic reaction mechanisms, such as emission from a fast moving source that originates from fragmentation of the target nucleus. However, fast moving sources are perhaps more naturally associated with reactions at much higher incident nucleon energies of several $\mathrm{GeV}$, and it is debatable how the mechanism extrapolates towards lower incident energies. A much simpler process, which is in principle also able to account for the observed effect, is the participation of sequential decay from inelastically excited states in the target nucleus which decay by $\alpha$-particle emission. This type of reaction mechanism is very often encountered in $(p, p \alpha)$ investigations in the incident energy range currently of interest, where it merely serves to interfere with the knockout reaction of primary concern.

Thus sequential $\alpha$-particle decay from low-lying inelastic excitations seems to be a serious contender as a source of yield in inclusive $(p, \alpha)$ reactions at emission energies down to those normally associated with a compound system. Studies of the $(p, p \alpha)$ reaction for various target nuclei in a range of kinematic conditions, which are very different from values of interest to knockout investigations, need to be explored.

This work was performed with funding from the South African National Research Foundation (NRF). The financial support is gratefully acknowledged.

\section{References}

1. D.S. Ginger, K. Kwiatkowski, G. Wang, W.-c. Hsi, S. Hudan, E. Cornell, R.T. de Souza, V.E. Viola, and R.G. Korteling, Phys. Rev. C 78 (2008) 034601.

2. E. Gadioli and P.E.Hodgson, Pre-equilibrium Nuclear Reactions (Oxford University Press, New York, 1991). 


\section{$\mathrm{CNR} * 11$}

3. A.J. Koning, S. Hilaire, and M.C. Duijvestijn, TALYS-1.0, Proceedings of the International Conference on Nuclear Data for Science and Technology, April 22-27, 2007, Nice, France, editors O. Bersillon, F. Gunsing, E. Bauge, R. Jacqmin, and S. Leray, EDP Sciences, 2008, p. 211-214.

4. A.J. Koning and M.C. Duijvestijn, Nucl. Phys. A 744 (2004) 1576.

5. R. Bevilacqua, PhD Thesis, Uppsala University (2011).

6. A.A. Cowley, G.J. Arendse, J.W. Koen, W.A. Richter, J.A. Stander, G.F. Steyn, P. Demetriou, P.E. Hodgson, and Y. Watanabe. Phys. Rev. C 54 (1996) 778.

7. A. Budzanowski, M. Fidelus, D. Filges, F. Goldenbaum, H. Hodde, L. Jarczyk, B. Kamys, M. Kistryn, St. Kistryn, St. Kliczewski, A. Kowalczyk, E. Kozik, P. Kulessa, H. Machner, A. Magiera, B. Piskor-Ignatowicz, K. Pysz, Z. Rudy, R. Siudak, and M. Wojciechowski, Phys. Rev. C 80 (2009) 054604.

8. A.A. Cowley, G.J. Arendse, G.F. Steyn, J.A. Stander, W.A. Richter, S.S. Dimitrova, P. Demetriou, and P.E. Hodgson, Phys. Rev. C 55 (1997) 1843.

9. R. Bevilacqua, S. Pomp, V. Simutkin, M. Hayashi, S. Hirayama, Y. Naitou, Y. Watanabe, U. Tippawan, M. Tesinsky, G. Ban, J.-L. Lecouey, N. Marie and Q. Hamel, Nuclear Instrument and Methods in Physics Research A 646 (2011) 100.

10. P.G. Roos, N.S. Chant, A.A. Cowley, D.A. Goldberg, H.D. Holmgren, and R. Woody, Phys. Rev. C 15 (1977) 69.

11. J. Mabiala, A.A. Cowley, S.V. Förtsch, E.Z. Buthelezi, R. Neveling, F.D. Smit, G.F. Steyn, and J.J. van Zyl, Phys. Rev. C 79 (2009) 054612.

12. T.A. Carey, P.G. Roos, N.S. Chant, A. Nadasen, and H.L. Chen, Phys. Rev. C 29 (1984) 1273.

13. T.Yoshimura, A. Okihana, R.E.Warner, N. S. Chant, P.G. Roos, C. Samanta, S. Kakigi, N. Koori, M. Fujiwara, N. Matsuoka, K. Tamura, E. Kubo, and K. Ushiro, Nucl. Phys. A641 (1998) 3 .

14. A. Budzanowski, M. Fidelus, D. Filges, F. Goldenbaum, H. Hodde, L. Jarczyk, B. Kamys, M. Kistryn, St. Kistryn, St. Kliczewski, A. Kowalczyk, E. Kozik, P. Kulessa, H. Machner, A. Magiera, B. Piskor-Ignatowicz, K. Pysz, Z. Rudy, R. Siudak, and M. Wojciechowski, Phys. Rev. C 78 (2008) 024603.

15. A. Budzanowski, M. Fidelus, D. Filges, F. Goldenbaum, H. Hodde, L. Jarczyk, B. Kamys, M. Kistryn, St. Kistryn, St. Kliczewski, A. Kowalczyk, E. Kozik, P. Kulessa, H. Machner, A. Magiera, B. Piskor-Ignatowicz, K. Pysz, Z. Rudy, R. Siudak, and M. Wojciechowski, Phys. Rev. C 82 (2010) 034605. 